\title{
BMJ Open Vision impairment and differential access to eye health services in Aotearoa New Zealand: protocol for a scoping review
}

\author{
Jaymie Tingkham Rogers (D) , ${ }^{1}$ Joanna Black (D) , ${ }^{1}$ Matire Harwood (D) , \\ Ben Wilkinson (D) , ${ }^{3}$ Iris Gordon (D) , ${ }^{4}$ Jacqueline Ramke (D) ${ }^{1,4}$
}

To cite: Rogers JT, Black J, Harwood M, et al. Vision impairment and differential access to eye health services in Aotearoa New Zealand: protocol for a scoping review. BMJ Open 2021;11:e048215. doi:10.1136/ bmjopen-2020-048215

- Prepublication history and additional supplemental material for this paper are available online. To view these files, please visit the journal online. (http://dx.doi.org/10.1136/ bmjopen-2020-048215).

Received 23 December 2020 Accepted 04 August 2021

\section{Check for updates}

(c) Author(s) (or their employer(s)) 2021. Re-use permitted under CC BY-NC. No commercial re-use. See rights and permissions. Published by BMJ.

${ }^{1}$ School of Optometry \& Vision Science, The University of Auckland Faculty of Medical and Health Sciences, Auckland, New Zealand

${ }^{2}$ General Practice \& Primary Health Care, The University of Auckland School of Population Health, Auckland, New Zealand ${ }^{3}$ Department of Ophthalmology, Auckland District Health Board, Auckland, New Zealand ${ }^{4}$ International Centre for Eye Health, London School of Hygiene \& Tropical Medicine, London, UK

Correspondence to Ms Jaymie Tingkham Rogers; j.rogers@auckland.ac.nz

\section{ABSTRACT}

Introduction In Aotearoa New Zealand, Māori and Pacific people experience worse health outcomes compared with other New Zealanders. No population-based eye health survey has been conducted, and eye health services do not generate routine monitoring reports, so the extent of eye health inequality is unknown. This information is required to plan equitable eye health services. Here we outline the protocol for a scoping review to report the nature and extent of the evidence reporting vision impairment, and the use of eye health services by ethnicity in New Zealand. Methods and analysis An information specialist will conduct searches on MEDLINE and Embase, with no limit on publication dates or language. We will search the grey literature via websites of relevant government and service provider agencies. Reference lists of included articles will be screened. Observational studies will be included if they report the prevalence of vision impairment, or any of the main causes (cataract, uncorrected refractive error, macular degeneration, glaucoma or diabetic retinopathy) or report the use of eye health services in New Zealand among people of any age. Two authors will independently review titles, abstracts and full-text articles, and complete data extraction. Overall findings will be summarised using descriptive statistics and thematic analysis, with an emphasis on disaggregation by ethnicity where this information is available.

Ethics and dissemination Ethical approval has not been sought as our review will only include published and publicly accessible data. We will publish the review in an open access peer-reviewed journal. We anticipate the findings will be useful to organisations and providers in New Zealand responsible to plan and deliver eye care services, as well as stakeholders in other countries with differential access to eye care.

Registration details The protocol has been registered with Open Science Framework (https://osf.io/yw7xb).

\section{INTRODUCTION}

Rationale

The recent Lancet Global Health Commission on Global Eye Health defined eye health as 'maximised vision, ocular health, and functional ability, thereby contributing to overall health and wellbeing, social inclusion, and quality of
Strengths and limitations of this study

- The broad scope of this review will result in the first synthesis to date on the extent of the evidence on vision impairment, its main causes and use of eye health services across ethnicity groups in New Zealand.

- The search will be performed by an information specialist, and screening and data extraction will be performed in duplicate.

- We anticipate limited information on some causes of vision impairment, and inconsistent disaggregation of outcomes by ethnicity.

life'. ${ }^{1}$ Eye health services are then considered any service which contributes to this broad definition of eye health. The need for accessible eye health services is large and increasing. Globally, an estimated 43 million people were blind and 295 million people had moderate or severe vision impairment in $2020 .^{2}$ In highincome countries, including Aotearoa New Zealand (hereafter referred to as New Zealand), the main causes of blindness and moderate or severe vision impairment (collectively referred to as vision impairment) are cataract, macular degeneration, glaucoma, uncorrected refractive error and diabetic retinopathy. ${ }^{3}$ Most people with vision impairment are older adults, however, diabetic retinopathy is the leading cause of vision impairment in the working age group. ${ }^{4}$ Diabetic retinopathy is projected to be an increasingly common cause of vision impairment in the coming decades due to the rising prevalence of diabetes. $^{5}$

Although some countries are striving to reduce health inequalities, ${ }^{6}$ achieving equitable health outcomes is an intractable challenge. ${ }^{7}$ In many countries, people who are Indigenous, living with socioeconomic disadvantage and marginalised communities face barriers to accessing healthcare. ${ }^{8}$ Consequently, systemic 
and chronic health conditions are more prevalent among these people. ${ }^{9}$ They also tend to have higher rates of vision impairment. ${ }^{1011}$ For example, in Australia the prevalence of cataract is higher among Indigenous people compared with non-Indigenous Australians, reflecting lower access to eye health services. ${ }^{812}$

Māori, the Indigenous people of New Zealand, are one of six main ethnicity groups (defined by Statistics New Zealand as 'a cultural group a person identifies with or has a sense of belonging to'). ${ }^{13}$ In the 2018 census, $70 \%$ of New Zealanders identified with at least one European ethnicity, $17 \%$ identified as Māori, $8 \%$ identified with at least one Pacific peoples' ethnicity, $15 \%$ identified as Asian, 2\% identified as Middle Eastern/Latin American/ African and $1 \%$ identified as other ethnicity. ${ }^{14}$

Inequities in health and variations between ethnicity groups in the prevalence of systemic diseases have been reported in New Zealand. ${ }^{15}$ The health gap is persistent between Māori and non-Māori. ${ }^{16}$ Chronic conditions such as diabetes, cardiovascular disease and chronic obstructive pulmonary disease are more prevalent among Māori compared with other New Zealanders. ${ }^{17} 18$ Māori have a higher need for renal replacement therapy ${ }^{15}$ and a 30\% higher risk of developing a cardiovascular event compared with European New Zealanders. ${ }^{19} 1516171815$

Inequities in eye health are well-documented in several high-income countries. ${ }^{12} 2021$ In New Zealand, the extent of inequity in eye health is largely unknown. New Zealand has never had a population-based eye health survey. A systematic review has been conducted on diabetic retinopathy prevalence and services, ${ }^{22}$ but synthesis of information on other causes of vision impairment has not been undertaken. This information would assist decisionmakers to plan equitable eye health services.

The aim of this scoping review is to summarise the nature and extent of evidence in New Zealand on:

1. The distribution of vision impairment and its major causes by ethnicity.

2. Differential access to eye health services by ethnicity.

As there is no New Zealand-specific information available on the main causes of vision impairment, we will assess the evidence on main causes in high-income countries. ${ }^{3}$ We chose to undertake a scoping review rather than a systematic review, as we anticipate that the available evidence will be heterogenous. ${ }^{23}$

\section{METHODS AND ANALYSIS}

We will follow the methodological steps for scoping reviews outlined by Arksey and O'Malley. ${ }^{23}$ Our team includes researchers (JB, MH, JR) with experience in conducting scoping reviews including on service delivery models to address inequities in eye health. ${ }^{2425}$

\section{Objectives/scoping review questions}

To achieve our aim, we will answer the following questions:

1. What is the nature and extent of the available evidence on vision impairment in New Zealand?
2. What is the available evidence on the prevalence of the major causes of vision impairment in New Zealand?

3. How and in what ways is vision impairment and its major causes distributed across ethnicity groups?

4. What is the available evidence on differential access to eye health services for the major causes of vision impairment by ethnicity?

\section{Protocol and registration}

The protocol for this scoping review is reported according to the relevant items of the Preferred Reporting Items for Systematic reviews and Meta-Analyses (PRISMA) extension for Scoping Reviews checklist (online supplemental annex 1). ${ }^{26}$ The protocol has been registered with Open Science Framework.

\section{Patient and public involvement}

There is no patient or public involvement as our review will only include published and publicly accessible data.

\section{Eligibility criteria}

We will include studies that meet the following criteria:

\section{Context}

Studies will be included if they report outcomes among residents of New Zealand (whether disaggregated by ethnicity or not), or attendees at New Zealand health facilities (regardless of facility size, public/private sector or level of care). Multi-country studies will be included if the results are reported separately for New Zealand.

\section{Types of studies}

Observational study types such as cross-sectional, casecontrol and consecutive case series will be included. Nonconsecutive cases series will be excluded. Research letters and grey literature, such as District Health Board (DHB) reports will be included, if they report data for at least one of our outcomes of interest. Editorials and conference abstracts will be excluded. We will have no time limit or language restrictions. Only studies where the full article is available will be included. We will use the University of Auckland's comprehensive inter-library loan service to retrieve articles not readily available.

\section{Participants}

We will include studies of any population group resident in New Zealand. There will be no age or gender restriction.

\section{Concept/outcomes}

We will include studies that report at least one of:

- The prevalence of vision impairment.

- The prevalence of cataract, uncorrected refractive error, macular degeneration, glaucoma or diabetic retinopathy.

- The prevalence of vision impairment due to cataract, uncorrected refractive error, macular degeneration, glaucoma or diabetic retinopathy. 
- Attendance at eye health service such as ophthalmology services, optometric services and eye health screening programmes (eg, diabetic retinal screening, children vision screening).

- Rates of treatment for cataract, uncorrected refractive error, macular degeneration, glaucoma or diabetic retinopathy.

We will include studies which report these outcomes by person. Studies which only report the outcomes by eye or by eye health service visit will be excluded.

\section{Search}

Published literature search

We will search MEDLINE and Embase using search strategies developed by a Cochrane Eyes and Vision Information Specialist (IG). Our search strategy used on MEDLINE is included in online supplemental annex 2. We will apply a backward and forward snowball citation approach to identify potentially relevant studies, ${ }^{27}$ by examining reference lists of all included articles (backward) and examining studies which have cited our included articles (forward).

\section{Grey literature search}

We will include grey literature that reports data for at least one of our outcomes. General search terms will be used to identify eligible information within each website. Relevant links within documents to other sources of information will be pursued. A single reviewer will perform the search and identify eligible data, with verification from a second reviewer.

Using Google search engine, separate searches will be performed across:

- New Zealand government websites such as Ministry of Health and DHBs.

- Professional associations such as New Zealand Association of Optometry and the Royal Australian and New Zealand College of Ophthalmologists.

- Non-profit organisations and charitable trusts such as Blind Low Vision New Zealand and Macular Degeneration New Zealand.

We will limit our search to the first 20 items of Google search engines.

Google search terms will include:

- "vision", "eye", "eye health", "eye service", "vision tests" and "vision screening".

- "cataract", "uncorrected refractive error", "macular degeneration", "glaucoma" or "diabetic retinopathy".

In addition, field experts and key stakeholders will be contacted to share our list of included studies and request they identify further potentially relevant studies for consideration in the review.

\section{Study selection}

Covidence systematic review software will be used for screening (Veritas Health Innovation, Melbourne, Australia. Available at: www.covidence.org). Two reviewers will independently screen the title and abstract of identified studies to exclude publications that clearly do not meet the inclusion criteria. The full-text article will be retrieved for review (via the University of Auckland Library) if the citation seems potentially relevant. Any discrepancies between the reviewers will be resolved by discussion and a third reviewer will be consulted if necessary. A PRISMA flow diagram will be completed to summarise the study selection process.

\section{Data charting process}

A custom form will be developed in Excel for data charting. The form will be piloted on three studies and required amendments agreed by consensus. As we anticipate a broad scope of studies, the data charting process will be iterative and the data charting form will be amended as required. Each included study will be charted independently by two reviewers. Any discrepancies between the reviewers will be resolved by discussion, and a third reviewer will be consulted if necessary. We plan to contact study authors in the case of unclear information and will make up to three attempts by email.

\section{Data items}

The following data items will be collected during the data charting process:

1. Source characteristics:

a. Published data-author(s), year of publication, title, journal and study design.

b. Grey literature-author (organisation, eg, Ministry of Health), year of publication, source website (eg, government/non-government organisation), type of literature (report, thesis, technical report, statistic, other).

2. Study characteristics: year(s) of data collection, sample size, age group of study population, demographics of study population such as gender and ethnicity. Geographic area (eg, city, district) and study setting (eg, facility level).

3. Outcomes as outlined above. We will extract all outcomes at the aggregate level, as well as disaggregated by ethnicity, gender, DHB and area level deprivation wherever available.

\section{Synthesis of results}

We will summarise findings narratively and in tables. Information for each outcome will be disaggregated by cause of impairment, ethnicity, age, geographic region and area level deprivation where these are available. ${ }^{28}$

Where possible, we will use Statistics New Zealand level 2 main categories for ethnicity (European, Māori, Pacific people, Asian and Middle Eastern/Latin American/ African), ${ }^{13}$ and otherwise report according to information provided by authors.

Where possible, we will use the International Classification of Diseases 11th classification (ICD-11) categories of vision impairment, based on presenting visual acuity in the better eye, that is, mild vision impairment is visual acuity of $6 / 12$ or worse to $6 / 18$ inclusive; moderate vision impairment is visual acuity worse than $6 / 18$ to $6 / 60$ inclusive; severe vision 
impairment is visual acuity worse than $6 / 60$ to $3 / 60$ inclusive and blindness is visual acuity worse than $3 / 60 .{ }^{29}$

We will share our synthesis of the results with the field experts and key stakeholders engaged during the search process, to get feedback on our summary of results. ${ }^{23}$

\section{ETHICS AND DISSEMINATION}

Ethical approval has not been sought as our review will only include published and publicly accessible data. We will publish the review in an open access peer-reviewed journal. We anticipate the findings will be useful to organisations and providers in New Zealand responsible to plan and deliver eye care services, as well as stakeholders in other countries with differential access to eye care.

\section{Collaborators N/A}

Contributors JTR drafted the protocol with suggestions from JR, JB, MH, IG and BW who reviewed the protocol and provided feedback on the draft. IG constructed the search. The final version of the protocol was approved by all authors.

Funding The authors have not declared a specific grant for this research from any funding agency in the public, commercial or not-for-profit sectors.

Disclaimer Award/grant number is not applicable. This research received no specific grant from any funding agency in the public, commercial or not-for-profit sectors. JTR is a recipient of the University of Auckland Senior Health Research Scholarship. JR's appointment at the University of Auckland is funded by the Buchanan Charitable Foundation, New Zealand.

\section{Competing interests None declared.}

Patient consent for publication Not required.

Provenance and peer review Not commissioned; externally peer reviewed.

Supplemental material This content has been supplied by the author(s). It has not been vetted by BMJ Publishing Group Limited (BMJ) and may not have been peer-reviewed. Any opinions or recommendations discussed are solely those of the author(s) and are not endorsed by BMJ. BMJ disclaims all liability and responsibility arising from any reliance placed on the content. Where the content includes any translated material, BMJ does not warrant the accuracy and reliability of the translations (including but not limited to local regulations, clinical guidelines, terminology, drug names and drug dosages), and is not responsible for any error and/or omissions arising from translation and adaptation or otherwise.

Open access This is an open access article distributed in accordance with the Creative Commons Attribution Non Commercial (CC BY-NC 4.0) license, which permits others to distribute, remix, adapt, build upon this work non-commercially, and license their derivative works on different terms, provided the original work is properly cited, appropriate credit is given, any changes made indicated, and the use is non-commercial. See: http://creativecommons.org/licenses/by-nc/4.0/.

\section{ORCID iDs}

Jaymie Tingkham Rogers http://orcid.org/0000-0003-2415-8198

Joanna Black http://orcid.org/0000-0002-5100-8796

Matire Harwood http://orcid.org/0000-0003-1240-5139

Ben Wilkinson http://orcid.org/0000-0002-2629-7063

Iris Gordon http://orcid.org/0000-0001-8143-8132

Jacqueline Ramke http://orcid.org/0000-0002-5764-1306

\section{REFERENCES}

1 Burton MJ, Ramke J, Marques AP, et al. The Lancet global health Commission on global eye health: vision beyond 2020. Lancet Glob Health 2021;9:e489-551.

2 Bourne R, Adelson J, Flaxman S. Trends in prevalence of blindness and distance and near vision impairment over 30 years and contribution to the global burden of disease in 2020. Lancet Glob Health 2020. doi:10.2139/ssrn.3582742

3 Bourne RRA, Jonas JB, Bron AM, et al. Prevalence and causes of vision loss in high-income countries and in eastern and central Europe in 2015: magnitude, temporal trends and projections. $\mathrm{Br} J$ Ophthalmol 2018;102:575-85.
4 Wong TY, Sabanayagam C. Strategies to tackle the global burden of diabetic retinopathy: from epidemiology to artificial intelligence. Ophthalmologica 2020;243:9-20.

5 Yau JWY, Rogers SL, Kawasaki R, et al. Global prevalence and major risk factors of diabetic retinopathy. Diabetes Care 2012;35:556-64.

6 Martin S, Siciliani L, Smith P. Socioeconomic inequalities in waiting times for primary care across ten OECD countries. Soc Sci Med 2020;263:113230.

7 Ministry of Health. Achieving equity in health outcomes: highlights of important national and international papers. Wellington, New Zealand: Ministry of Health, 2018.

8 Taylor HR, Xie J, Fox S, et al. The prevalence and causes of vision loss in Indigenous Australians: the National Indigenous eye health survey. Med J Aust 2010;192:312-8.

$9 \mathrm{Gu}$ Y, Warren J, Kennelly J, et al. Cardiovascular disease risk management for Māori in New Zealand general practice. J Prim Health Care 2014;6:286-94.

10 Foreman J, Keel S, van Wijngaarden P, et al. Prevalence and causes of visual loss among the Indigenous peoples of the world: a systematic review. JAMA Ophthalmol 2018;136:567-80.

11 Foreman J, Xie J, Keel S, et al. The prevalence and causes of vision loss in Indigenous and non-Indigenous Australians: the National eye health survey. Ophthalmology 2017;124:1743-52.

12 Kelaher M, Ferdinand A, Taylor H. Access to eye health services among Indigenous Australians: an area level analysis. BMC Ophthalmol 2012;12:51.

13 Stats NZ Tatauranga Aotearoa. Census ethnic groups dataset, 2018. Available: https://www.stats.govt.nz/information-releases/2018census-ethnic-groups-dataset [Accessed Dec 2020].

14 Stats NZ Tatauranga Aotearoa. Census data, 2018. Available: https:// www.stats.govt.nz/tools/2018-census-place-summaries/newzealand\#ethnicity-culture-and-identity [Accessed Dec 2020].

15 Atlantis E, Joshy G, Williams M. Diabetes among Māori and other ethnic groups in New Zealand. In: Diabetes mellitus in developing countries and underserved communities, 2017: 165-90.

16 Ferdinand A, Lambert M, Trad L, et al. Indigenous engagement in health: lessons from Brazil, Chile, Australia and New Zealand. Int J Equity Health 2020;19:1-12.

17 Sheridan NF, Kenealy TW, Connolly MJ, et al. Health equity in the New Zealand health care system: a national survey. Int J Equity Health 2011;10:45.

18 Ellison-Loschmann L, Pearce N. Improving access to health care among New Zealand's Maori population. Am J Public Health 2006;96:612-7.

19 Kenealy T, Elley CR, Robinson E, et al. An association between ethnicity and cardiovascular outcomes for people with type 2 diabetes in New Zealand. Diabet Med 2008;25:1302-8.

20 Aljied R, Aubin M-J, Buhrmann R, et al. Prevalence and determinants of visual impairment in Canada: cross-sectional data from the Canadian longitudinal study on aging. Can J Ophthalmol 2018;53:291-7.

21 Yip JLY, Luben R, Hayat S, et al. Area deprivation, individual socioeconomic status and low vision in the EPIC-Norfolk eye study. J Epidemiol Community Health 2014;68:204-10.

22 Ramke J, Jordan V, Vincent AL, et al. Diabetic eye disease and screening attendance by ethnicity in New Zealand: a systematic review. Clin Exp Ophthalmol 2019;47:937-47.

23 Arksey H, O'Malley L. Scoping studies: towards a methodological framework. Int J Soc Res Methodol 2005;8:19-32.

24 Burn H, Hamm L, Black J, et al. Eye care delivery models to improve access to eye care for Indigenous peoples in high-income countries: a scoping review. BMJ Glob Health 2021;6:e004484.

25 Hamm LM, Black J, Burn H, et al. Interventions to promote access to eye care for non-Indigenous, non-dominant ethnic groups in high-income countries: a scoping review protocol. BMJ Open 2020;10:e033775.

26 Tricco AC, Lillie E, Zarin W, et al. PRISMA extension for scoping reviews (PRISMA-ScR): checklist and explanation. Ann Intern Med 2018;169:467-73.

27 Jalali S, Wohlin C. Systematic literature studies: database searches vs. backward snowballing. Paper presented at: International Symposium on Empirical Software Engineering and Measurement, 2012.

28 Atkinson J, Salmond C, Crampton P. NZDep2013 index of deprivation. Wellington: Department of Public Health, University of Otago, Wellington, 2014. https://www.otago.ac.nz/wellington/ otago069936.pdf

29 WHO. International classification of disease 11 vision impairment including blindness, 2018. Available: https://icd.who.int/browse11/ I-m/en\#/http\%3a\%2f\%2fid.who.int\%2ficd\%2fentity\%2f30317704 [Accessed Dec 2020]. 